\title{
The Load Current Control of Inductively Coupled Power Transfer System Based on Neural Network for AUV
}

\author{
Y.Q. Yuan \\ Shenyang Institute of Automation \\ Chinese Academy of Sciences \\ Shenyang
}

\author{
K. Wang \\ University of Chinese Academy of Sciences \\ Shenyang Institute of Automation \\ Chinese Academy of Sciences \\ Shenyang
}

\begin{abstract}
Autonomous Underwater Vehicle non-contact charging system using a separate structure, When the technology is used in the underwater environment, the inductive coupled power transmission system will produce a gap and eccentricity under the influence of the water shock, causing the coupling state changed, which will affect the output power. This paper presents a control algorithm based on neural network system that compensate the system output power by adding a DC/DC switching according to the changes of the magnetic coupling state, in order to achieve a stable output voltage.
\end{abstract}

Keywords-inductive coupled power transfer; coupling; neural network; power compensation

\section{INTRODUCTION}

Autonomous Underwater Vehicle(AUV)is a kind of robot which depend on their own energy to accomplish specific underwater tasks.AUV have broad application prospects and potential applications in the marine industry, marine environmental science and military fields[1].In recent years, inductive power transmission technology has got fast development, AUV can dock the seabed station to supplement energy, this method can not only increase the AUV underwater time but also improve efficiency, which can also enhance the sneak's covert.

Due to the use of inductively coupled power transfer system loosely coupled transformer, the primary and secondary side of the coupler is completely isolated. Meanwhile in the deep sea environment, the system need to be docked by complete automation. To improve the life of the system, which needs a larger redundancy docking structure. The primary and secondary systems side will produce eccentric and clearance under the impact of water, which will have a great effect on the coupling coefficient and lead to instability in output power. Literature [2] proposed a frequency locked loop control strategy based on control of AUV non-contact charging system, but it does not validate the control effect. Literature [3] used the PID controller

\author{
Y.H. Liao \\ University of Chinese Academy of Sciences \\ Shenyang Institute of Automation \\ Chinese Academy of Sciences \\ Shenyang
}

full-bridge circuit to adjust the phase angle to achieve a constant current load, because of ICPT's nonlinear, time-varying uncertainties and other factors, it is difficult to establish a precise mathematical model, PID controller is difficult to achieve the desired control effect. Literature [4] used fuzzy control algorithm to control the pulse duty cycle to get a stable output of the system, but longer reaction time of this algorithm is not suitable for an underwater environment.

This paper proposes the control algorithm based on neural network, adding DC / DC boost circuit and changing the duty cycle of the switching circuit to achieve dynamic compensation for power to get a stable output voltage. This method guarantee the system output voltage constant, which overcome the eccentric and clearance produced by the primary and secondary core because of the water's effect.

\section{CHARACTERISTICS OF ICPT SySTEM POWER TRANSMISSION}

Figure 1 shows a SS-type compensation ICPT topology.



FIGURE I. SS-TYPE COMPENSATION ICPT TOPOLOGY.

The system consists of two parts:(1) consist of the power converter and the primary transmission coil, the former is to provide a constant current to the primary coil.(2)consist of the rectifier filter circuit and the secondary coil, which will rectifier the coupling voltage for the DC load. In order to achieve maximum power output, the system must ensure that the original side and the secondary side are in a resonant state, the secondary resonant capacitor Cs must meet[5]: 


$$
\omega^{2} L_{s} C_{s}=1
$$

The total impedance of the system is equivalent as:

$$
Z_{\text {total }}=j \omega L_{p}+\frac{1}{j \omega C_{p}}+R_{p}+\frac{\omega^{2} M^{2}}{Z_{s}}
$$

In order to reduce the reactive power components of the overall system, to achieve overall system drive resistive circuit, the primary resonance compensation capacitor selection needs to meet the imaginary part is zero, which can have a value of $\mathrm{Cp}$

$$
C_{p}=\frac{1}{\omega^{2} L_{P}}
$$

SS type ICPT system available output power is:

$$
P_{\text {out }}=\frac{\omega^{2} M^{2} V_{p-v}{ }^{2} R}{\left(\omega^{2} M^{2}+R_{p} R\right)^{2}}
$$

From the formula $2-4$, we can know when the resonance frequency and the primary voltage load is constant, output power of the system relate to the value of the mutual inductance. As the literature [6] shows, the value of $\mathrm{M}$ can be easily obtained, it is feasible to change the duty cycle of the boost switch according to the dynamics of M's value to maintain a stable output voltage. When the voltage of the load is stable, the output power of the load is stable. By the formula (4) known the voltage of the load is [7]

$$
U=\frac{\omega M V_{p-v} R}{\left(\omega^{2} M^{2}+R_{p} R\right)}
$$

In the deep sea environment, the ICPT system need automatic docking, it is necessary to reserve some space mission to improve the life of system. When the core is under the impact of the sea, the eccentricity and the gap will change. The gap has an impact on the magnetic length and equivalent cross-sectional are. The coupling coefficient will change with the gap's diversification. The above analysis shows that the system's output power is determined by the mutual inductance and the resonance frequency. When the resonance frequency of the system is fixed, the system's output power is determined by the mutual inductance. In the underwater, he complex water environment will make the mutual inductance continue changing within a certain range, which can not guarantee the output power is constant. Especially when the current exceeds the tolerance range of the load, the load will be damaged. The curves of coupling coefficient vary with gap and eccentric are shown in figures 2 and 3.

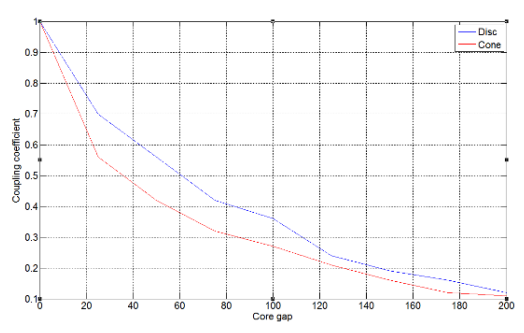

FIGURE II. CURVE OF COUPLING COEFFICIENT VARYING WITH CORE GAP.

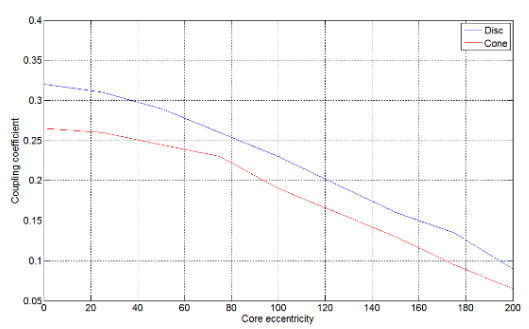

FIGURE III. CURVE OF COUPLING COEFFICIENT VARYING WITH CORE ECCENTRICITY.

To ensure a constant voltage across the load, you can add a booster circuit to boost the primary power source, Reference [8] show that the initial voltage $\mathrm{U}_{\mathrm{o}}$ and the target voltage $\mathrm{U}_{\mathrm{i}}$ satisfies the relationship:

$$
M=\frac{U_{o}}{U_{i}}=\frac{1}{1-D_{c}}
$$

By the formula (7) formula (8) duty cycle of boost switch to maintain a constant load terminal voltage can be obtained:

$$
\Delta=\frac{U R \omega^{2} M^{2}+R_{p} R^{2} \omega M-V_{p-v} \omega^{2} M^{2} R}{U M \omega+U R_{p} R}
$$

\section{III.DESIGN OF CONSTANT CURRENT CONTROL SYSTEM BASED ON NEURAL NETWORK}

\section{A. Adaptive Control Strategy Based on Neural Network}

From the forenamed, when the core is under the impact of the sea, the eccentricity and the gap will change which will make the mutual inductance changed. Because of ICPT's nonlinear, time-varying uncertainties and other factors, it is difficult to establish a precise mathematical model. The neural networks does not depend on accurate mathematical model of the controlled object. The neural network is extremely easy to deal with high-dimensional, nonlinear, uncertainty control problems. This paper is based on neural networks to design load current control system, as is shown in Figure 4.

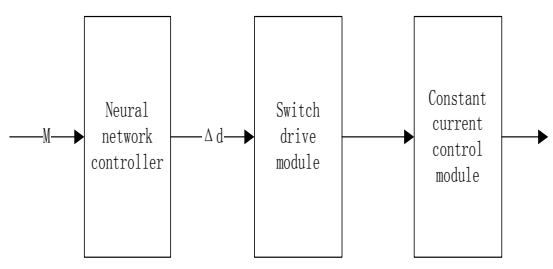

FIGURE IV. SYSTEM CONTROL BLOCK DIAGRAM. 
The system will measure the coil coupling coefficient, the neural network controller will ouput the duty cycle of DC/DC boost switch under this value, then driving constant current control module, achieving secondary output current constant.

\section{B. Design of BP Neural Network Controller}

BP neural network controller designing, including the numbers of layers of nodes, select and activate the relevant parameter setting functions and training methods between the layers, specifically including[9]

Determining the number of hidden layer: From the structural characteristics of neural networks, it can contain one or more hidden layer, in practical applications are generally a hidden layer can complete any nonlinear function mapping.

Determining the number of input layer nodes: The input layer accepts external input data, which depends on the actual number of nodes of the input vector dimension. In this paper the system output duty mainly make mutual inductance $\mathrm{k}$ as the input, therefore the number of nodes in the input layer is 1 .

Determining the number of output layer nodes: the $\mathrm{k}$ is used to control the duty cycle of the boost switch, therefore the output nodes of this article is 1.

Determining the number of hidden layer nodes: studies have shown that the hidden layer nodes is generally determined by input layer and output layer, generally satisfying the formula:

$$
q=\sqrt{p+r}+a, a \in[1,10]
$$

$\mathrm{q}$ is the hidden layer nodes, $\mathrm{p}$ is the input layer nodes, $\mathrm{r}$ is the output layer nodes, $a$ is a random number between 1 and 10 ,when the hidden layers is 5 can play a better training effect.

Choosing the activation function: Activation function occupies a pivotal position in BP neural network. In BP algorithm activation function must be continuous, differentiable. Usually using S-type activation function(including sigmoid function and double-cut tansig function) and pure linear function. If the final layer BP network activation function is sigmoid function, the output of the network will be limited to $[0,1]$;if the purelin function is selected, the output of the network can be any value. In this paper tansig function will be used between the input layer and the hidden layer, purelin function will be used between the hidden layer to the output layer.

Selecting training methods and parameters:

Training function: gradient descent BP arithmetic functions.

The learning rate is 0.05 .

Performance objectives is 0.001 .

Training maximum number of iterations is 1000 .

Neural network controller training mean square error performance function curve is shown in Figure 5.



FIGURE V. MSE PERFORMANCE FUNCTION.

\section{Simulation}

To analyze the effect of neural network controller, this paper based on Simulink platform to build SS type ICPT system model circuit to verify that the controller's effect when the output power is not constant because of the variation of the coupling coefficient. Model is shown in Figure 4, the parameters is shown in Table 1.

\section{TABLE I .SYSTEM PARAMETERS.}

\begin{tabular}{|c|c|}
\hline Parameters & Value \\
\hline DC voltage Vp-v/V & 48 \\
\hline Primary loop resonant inductor $\mathrm{Lp} / \mu \mathrm{H}$ & 100 \\
\hline Secondary loop resonant inductor $\mathrm{Ls} / \mu \mathrm{H}$ & 64 \\
\hline Mutual inductance $\mathrm{M} / \mu \mathrm{H}$ & 16 \\
\hline Primary loop coil resistance $\mathrm{Rp} / \Omega$ & 0.1 \\
\hline Secondary loop coil resistance $\mathrm{Rs} / \Omega$ & 0 \\
\hline Primary loop resonant capacitor $\mathrm{Cp} / \mu \mathrm{F}$ & 0.63 \\
\hline Secondary loop resonant capacitor $\mathrm{Cp} / \mu \mathrm{F}$ & 0.98 \\
\hline Load $\mathrm{R} / \Omega$ & 50 \\
\hline
\end{tabular}

The system controller make the coupling coefficient measured by voltammetry as an input, then the neural network controller will output the duty cycle of the DC/DC controller to boost the voltage source which will make the output power constant. The Figure 6 is when $\mathrm{K}$ is $0.25(\mathrm{~K}=0.3)$ the current change at the load. As seen from the figure the load current can be quickly adjusted to tend stable by $0.02 \mathrm{~s}$ and the control effect is very good.



FIGURE VI. CURRENT SIMULATION WAVEFORMS WHEN COUPING COUPLING COEFFICIENT IS 0.25 .

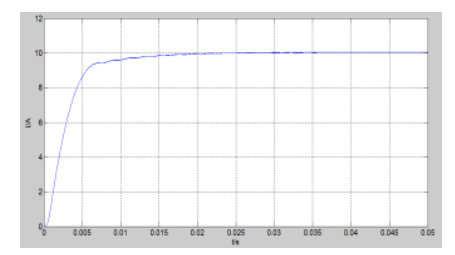

FIGURE VII. CURRENT SIMULATION WAVEFORMS WHEN COUPING COUPLING COEFFICIENT IS 0.3. 
Figure 8 is a coupling coefficient of the coupler in the range from 0.2 to 0.4 , the output current of the load corresponding to the change. We can see from the figure when the coupling coefficient is changed, the output current of the load change from 9.96A to $10.06 \mathrm{~A}$, the maximum overshoot of current is only $0.6 \%$ which can fully meet underwater application.

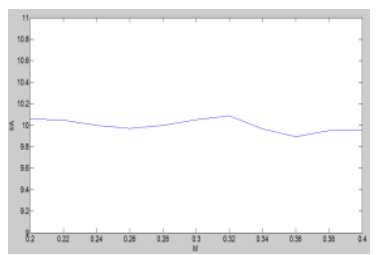

FIGURE VIII. THE CURRENT SIZE UNDER DIFFERENT COUPLING COEFFICIENTS.

\section{CONCLUSION}

In the underwater environment, when the inductive power transmission system of Autonomous Underwater Vehicle is under the impact of water, the mutual coefficient will change, which will make the output power deviate the normal value. This paper design a regulator system based on the neural networks, the system will control DC/DC switch duty cycle to ensure the output power of load is stable according to the coupling coefficient that obtained by measurement. The MATLAB simulation results show that the neural network controller has fast response, small overshoot adaptability, etc. This paper has some significance for the inductive power transmission system of Autonomous Underwater Vehicle.

\section{ACKNOWLEDGEMENTS}

Thank you for Liaoning Provincial Natural Science Foundation and National Natural Science Foundation of China.

\section{REFERENCES}

[1] MA Weifeng, HU Zhen. Current Researches and Development Trend on AUV[J].Fire Control and Command Control, 2008,06:10-13.

[2] TANG Fan, ZHANG Kehan, YAN Weisheng, SONG Baowei. Research on frequency control of contactless charging system of AUV[J].Ship Science and Technology, 2013,02:43-46+76.

[3] Thrimawithana D J, Madawala U K. A primary side controller for inductive power transfer systems[C]//Industrial Technology (ICIT), 2010 IEEE International Conference on. IEEE, 2010: 661-666.

[4] YANG Guang, TANG Houjun, BAI Yuliang. Design and Analysis of Fuzzy Constant Voltage Control for the CIPT Systems[J].Electrical Automation, 2013,04:41-43.

[5] Chwei-Sen Wang, Grant A Covic. Power transfer capability and bifurcation phenomena of loosely coupled inductive power transfer systems[J].IEEE Trans. Industrial Electronics , 2004,51(1):148-157.

[6] WANG Sujiao. Magnetic coupling coil mutual inductance $M$ measurement [J].Journal of Zhengzhou Railway Vocational College, 2002, 02:43-44.

[7] SUN Yue, XIA Chenyang, ZHAO Zhibin, ZHAI Yuan, YANG Fangxun. Analysis and optimization of voltage-system ICPT power transmission characteristics. Advanced Technology of Electrical Engineering and Energy, 2011,02:9-12+25.

[8] ZHOU Jing, WANG Xing. Application of voltage regulation system in contactless induction power transmission system[J].Chinese Journal of Power Sources, 2012,04:550-553.

[9] ZHANG Defeng. MATLAB neural network application design[M].Beijing: Mechanieal Industrial Publishing House,200 